\title{
Hedging under the Heston Model with Jump-to-Default
}

\author{
Peter Carr* \\ Wim Schoutens ${ }^{\dagger}$
}

First Version: 21st of September 2006

This Version: 21th of September 2007

*Bloomberg LP and Courant Institute of Mathematical Sciences, 731 Lexington Avenue, New York, NY 10022, U.S.A. E-mail: pcarr@nyc.rr.com

${ }^{\dagger}$ K.U.Leuven, Department of Mathematics, Celestijnenlaan 200 B, B-3001 Leuven, Belgium. E-mail: wim@schoutens. be 


\begin{abstract}
In this paper we will explain how to perfectly hedge under Heston's stochastic volatility model with jump to default, which is in itself a generalization of the Merton jump-to-default model and a special case of the Heston model with jumps. The hedging instruments we use to build the hedge will be as usual the stock and the bond, but also the Variance Swap (VS) and a Credit Default Swap (CDS). These instruments are very natural choices in this setting as the VS hedges against changes in the instantaneous variance rate, while the CDS protects against the occurrence of the default event.

First, we explain how to perfectly hedge a power payoff under the Heston model with jump to default. These theoretical payoffs play an important role later on in the hedging of payoffs which are more liquid in practice such as vanilla options. After showing how to hedge the power payoffs, we show how to hedge newly introduced Gamma payoffs and Dirac payoffs, before turning to the hedge for the vanillas. The approach is inspired by the Post-Widder formula for real inversion of Laplace Transforms. Finally, we will also show how power payoffs can readily be used to approximate any payoff only depending on the value of the underlier at maturity. Here, the theory of orthogonal polynomials comes into play and the technique is illustrated by replicating the payoff of a vanilla call option.
\end{abstract}




\section{Introduction}

Since its inception in 1993, the Heston stochastic volatility model [1] has received a great deal of attention from both practitioners and academics. It relaxes the constant volatility assumption in the classical Black-Scholes model by incorporating an instantaneous short term variance process. As such, many (though not all) smile and skew patterns can be built into volatility surfaces with a relatively small number of parameters. In this paper, we will explain how to perfectly hedge under Heston's stochastic volatility model with jump to default, which is in itself a generalization of the Merton jump-to-default model and a special case of the Heston model with jumps. The hedging instruments that we use to build the hedge include the underlying stock and a riskless asset (henceforth the bond) as usual. The hedge also involves a Variance Swap (VS) and a Credit Default Swap (CDS) of the same maturity as the target claim. These instruments are very natural choices in this setting. The VS hedges against changes in the instantaneous variance rate, while the CDS protects against the occurrence of the default event.

In this paper, we will first explain how to perfectly hedge a power payoff. These theoretical payoffs will play an important role later on in the hedging of more payoffs that are more liquid in practice such as vanilla options. After showing how to hedge the power payoff, we show how to hedge newly introduced Gamma and Dirac payoffs before turning to the hedge for the vanillas. Our approach is inspired by the Post-Widder [5] formula for real inversion of Laplace transforms. Finally, we will also show how power payoffs can readily be used to approximate any payoff only depending on the value of the underlier at maturity. Here, the theory of orthogonal polynomials comes into play and the technique is illustrated by a replication of the payoff of a vanilla call option.

This paper is organized as follows. In Section 2, we introduce the Heston model with jumpto-default. In Section 3, we show how to perfectly hedge power payoffs under this model. In the fourth section, we introduce Gamma and Dirac payoffs and elaborate on their use. In Section 5, we show how orthogonal polynomials can be used to approximate a target payoff. The final section summarizes the paper and suggests extensions.

\section{Heston Model with Jump-to-Default}

Let us briefly formalize the Heston model with Jump-to-Default (Heston+JtD). The dynamics of the stock price process $S=\left\{S_{t}, t \geq 0\right\}$ are very similar to the Black-Scholes setting.

$$
\mathrm{d} S_{t}=S_{t-}\left[(r-q) \mathrm{d} t+\sqrt{v_{t}} \mathrm{~d} W_{t}-\mathrm{d} N_{t}+\lambda \mathrm{d} t\right], \quad S_{0} \geq 0,
$$

where $r$ is as usual the short rate and $q$ the dividend yield. The instantaneous variance parameter is modeled as a mean-reverting square root stochastic process (also called CIR process), described by the following SDE:

$$
\mathrm{d} v_{t}=\kappa\left(\eta-v_{t}\right) \mathrm{d} t+\omega \sqrt{v_{t}} \mathrm{~d} \tilde{W}_{t}, \quad v_{0} \geq 0,
$$

where $W=\left\{W_{t}, t \geq 0\right\}$ and $\tilde{W}=\left\{\tilde{W}_{t}, t \geq 0\right\}$ are two correlated standard Brownian motions such that $\operatorname{Cov}\left[\mathrm{d} W_{t} \mathrm{~d} \tilde{W}_{t}\right]=\rho \mathrm{d} t$, and $N=\left\{N_{t}, t \geq 0\right\}$ is an independent Poisson process with intensity $\lambda>0$. The involved parameters are: initial variance, $v_{0}>0$, the mean reversion rate $\kappa>0$, the long run variance rate $\eta>0$, the volatility of the variance rate $\omega>0$, the jump-to-default intensity $\lambda>0$ and the correlation $\rho \in(-1,1)$. At the first time if any that the Poisson process jumps, the stock price jumps to the absorbing zero state. 
Before default occurs, the variance process is always positive and cannot reach zero if $2 \kappa \eta>\omega^{2}$. The latter is often referred to as the Feller condition. Under zero volatility of variance, the instantaneous variance rate is exponentially attracted to the level $\eta$, which explains why $\eta$ is called the long run variance rate. Typically, the correlation $\rho$ is negative, so that down-moves in the stock price tend to be coupled with up moves in volatility and vice versa. It is worthwhile mentioning that the variance process $v_{t}$ generates marginals given by a noncentral Chi-Squared distribution at each $t>0$, while the marginals generated by the volatility process $\sqrt{v_{t}}$ are always given by a Rayleigh distribution ([2]). In the analysis, an important role will be played by the characteristic function of the natural logarithm of the stock price:

$$
\phi\left(u, t ; S_{0}, v_{0}\right):=E\left[\exp \left(\mathrm{i} u \ln \left(S_{t}\right)\right) \mid S_{0}, v_{0}\right],
$$

where $\mathrm{i}$ is the imaginary unit. For our model, $\phi$ is given by

$$
\begin{aligned}
\phi\left(u, t ; S_{0}, v_{0}\right)=\quad & \exp \left(\mathrm{i} u\left(\ln S_{0}+(r-q) t\right)\right) \\
& \times \exp \left(\eta \kappa \omega^{-2}\left((\kappa-\rho \omega \mathrm{i} u-d) t-2 \log \left(\left(1-g \mathrm{e}^{-d t}\right) /(1-g)\right)\right)\right) \\
& \times \exp \left(\sigma_{0}^{2} \omega^{-2}(\kappa-\rho \omega \mathrm{i} u-d)\left(1-\mathrm{e}^{-d t}\right) /\left(1-g \mathrm{e}^{-d t}\right)\right) \\
& \times \exp (\lambda t(\mathrm{i} u-1)),
\end{aligned}
$$

where:

$$
\begin{aligned}
& d(u)=\sqrt{(\rho \omega u \mathrm{i}-\kappa)^{2}+\omega^{2}\left(\mathrm{i} u+u^{2}\right)} \\
& g(u)=(\kappa-\rho \omega \mathrm{i} u+d(u)) /(\kappa-\rho \omega \mathrm{i} u-d(u))
\end{aligned}
$$

\section{Hedging Under the Heston+JtD Model}

Consider a contingent claim whose payoff function has the form:

$$
G_{p}\left(S_{T}\right)=S_{T}^{p}, \text { for } p>0 .
$$

We will refer to these types of payoffs as power payoffs. The power $p$ can be any positive real number. Setting $p=0$ produces the payoff of a default-free Zero-Coupon Bond, while setting $p=1$ produces the terminal value of the underlying stock. In this section, we will show how to perfectly replicate power payoffs for all $p \geq 0$. Our replicating strategy involves dynamic trading in bonds, stocks, Credit Default Swaps (CDSs) (with zero recovery and notional 1) and Variance Swaps (VS) (based on squared relative returns and notional 1).

We note that for all $p>0$ the payoff is equal to one if $S_{T}=1$ and is zero in case of default $\left(S_{T}=0\right)$. Let us fix a $p>0$ and denote the time- $t$-price of this power payoff $G_{p}$ by $L_{t}$.

We then have that

$$
\begin{aligned}
L_{t}= & \exp (-r(T-t)) E_{Q}\left[S_{T}^{p} \mid S_{t}, v_{t}\right] \\
= & \exp (-r(T-t)) \phi\left(-\mathrm{i} p, T-t ; S_{t}, v_{t}\right) \\
= & \exp (-r(T-t)) S_{t}^{p} \exp \left(\eta \kappa \omega^{-2}\left(\kappa+\rho \omega p-d(-\mathrm{i} p)(T-t)-2 \log \left(\left(1-g(-\mathrm{i} p) \mathrm{e}^{-d(-\mathrm{i} p)(T-t)}\right) /(1-g(-\mathrm{i} p))\right)\right)\right) \\
& \quad \times \exp \left(v_{t} \omega^{-2}(\kappa+\rho \omega p-d(-\mathrm{i} p))\left(1-\mathrm{e}^{-d(-\mathrm{i} p)(T-t)}\right) /\left(1-g(-\mathrm{i} p) \mathrm{e}^{-d(-\mathrm{i} p) t}\right)\right) \\
& \quad \times \exp (\lambda(T-t)(p-1))
\end{aligned}
$$


which can be written in the form

$$
L_{t}=S_{t}^{p} \exp \left(C(T-t)+D(T-t) v_{t}\right),
$$

for some functions $C(s)$ and $D(s)$.

By applying Ito's formula for semi-martingales, one can show that the dynamics for the process $L=\left\{L_{t}, t \geq 0\right\}$ are given by

$$
\mathrm{d} L_{t}=L_{t-}\left(p \sqrt{v_{t}} \mathrm{~d} W_{t}+D(T-t) \omega \sqrt{v_{t}} \mathrm{~d} \tilde{W}_{t}-\mathrm{d} N_{t}+(* * *) \mathrm{d} t\right),
$$

where we do not specify the (complicated) $\mathrm{d} t$ term, because it is not needed in the derivation below. In what follows, we often write $(* * *)$ for the $\mathrm{d} t$ terms, but the reader is warned that the content of $(* * *)$ can change from one line to another.

Suppose that we are long one power claim for some fixed $p$. Our objective is to hedge away the randomness in the dynamics of $L_{t}$ by dynamically trading the underlying stock, a default-free bond, a VS, and a CDS. The last three hedge instruments are assumed to mature at the same time as the power claim.

We assume for the bond process the classical dynamics:

$$
\mathrm{d} B_{t}=r B_{t} \mathrm{~d} t
$$

The position in the bond will be determined last and will actually take care of all the $(* * *) \mathrm{d} t$-terms.

Noting (by reformulating Equation (1)) that:

$$
p L_{t-\sqrt{v_{t}}} \mathrm{~d} W_{t}=p L_{t-}\left(\mathrm{d} N_{t}-(r-q+\lambda) \mathrm{d} t+\frac{\mathrm{d} S_{t}}{S_{t-}}\right),
$$

we see that the correct number of shares needed for the hedge is

$$
M_{t-}^{S}=p L_{t-} / S_{t-} .
$$

Let us next analyze the behavior of a variance swap under this model. The floating leg of the variance swap which we will work with is based on the sum of the squares of the daily relative returns of the stock (not the squared log-returns). In the absence of a jump-to-default, the realization of this floating leg over a time interval $[t, T]$ is well approximated by $\int_{t}^{T} v_{t} \mathrm{~d} t$. In case a default occurs at some random time $\tau$, the pre-default realized variance of $\int_{t}^{\tau} v_{t} \mathrm{~d} t$ increases by $\left(\mathrm{d} S_{\tau} / S_{\tau-}\right)^{2}=1$. After the default time, the VS does not accumulate any more realized variance.

Conditioning on no default over $[t, T]$ the risk-neutral mean of the remaining realized variance is given by:

$$
\begin{aligned}
m\left(t, T ; v_{t}\right) & =E\left[\int_{t}^{T} v_{u} \mathrm{~d} u \mid v_{t}\right] \\
& =\eta(T-t)+\kappa^{-1}\left(v_{t}-\eta\right)(1-\exp (-\kappa(T-t))) \\
& =A(T-t)+B(T-t) v_{t} .
\end{aligned}
$$

for some functions $A(s)$ and $B(s)$. 
Since a jump-to-default in $[t, T]$ occurs with probability $1-\exp (-\lambda(T-t))$ and leads to a unit contribution to the floating leg of the VS, the instantaneous cash flow from being long one VS is:

$$
v_{t} \mathrm{~d} t+\mathrm{d} N_{t}-\lambda \mathrm{d} t-\mathrm{d} m\left(t, T ; v_{t}\right)
$$

This can hence be written as:

$$
(* * *) \mathrm{d} t+\mathrm{d} N_{t}-B(T-t) \sqrt{v_{t}} \omega \mathrm{d} \tilde{W}_{t} .
$$

This leads us to the number of VS needed in the hedge:

$$
M_{t}^{V S}=-L_{t-} D(T-t) / B(T-t)
$$

Note that the hedge position in VS can be entered into at zero cost.

Next, we calculate the number of CDS needed in the hedge. By taking a position of $L_{t-} D(T-$ $t) / B(T-t)$ variance swaps, we are exposed to a unit contribution to the realized variance in case of default. Furthermore, by the position in stock through Equation 2 and the necessity to take care of the $\mathrm{d} N_{t}$ term in Equation 1, we need to take a CDS position of

$$
M_{t}^{C D S}=L_{t-} D(T-t) / B(T-t)+(1-p) L_{t-} .
$$

Note that the hedge position in CDS can be entered into at zero cost. The investor who is long one CDS contract pays the constant CDS spread $\lambda \mathrm{d} t$ at each time $t$ before $\tau \wedge T$. In case the default occurs before $T$, this investor also receives a unit payment at the default time $\tau$. Hence at the default time $\mathrm{d} N_{t}=1$ and before then $\mathrm{d} N_{t}=0$. This means that the cash flow from the above CDS position is given by

$$
(* * *) \mathrm{d} t-L_{t-}(1-p+D(T-t) / B(T-t)) \mathrm{d} N_{t}
$$

The number of bonds needed at time $t$ in the hedge is just the difference between the theoretical value of the power claim and the total amount invested in stock, since the VS and CDS positions can be achieved at zero cost. Recall that the value of the power claim at time $t$ is given by $L_{t}$ and that the total stock position is given by $p L_{t-}$. As a result, we need to invest $(1-p) L_{t-}$ dollars in bonds. Note that in case $p=1$ the power claim is actually the stock, and hence the hedge is simply to short one share. Likewise in case $p=0$, the power claim is actually a bond and hence the hedge is simply to short one bond.

\section{Gamma and Dirac Payoffs}

In this section, we introduce two new payoff structures, which we refer to as the Gamma and Dirac payoffs. We will show how the results of the last section can be used to replicate these newly introduced payoffs. Furthermore, we will show how on the basis of these new payoffs, one can construct any path-independent payoff written only on the terminal stock price.

For technical reasons, we need to fix a lower barrier $H$, say at one percent of $S_{0}$, which the stock price cannot cross except through a jump-to-default. When stock prices can diffuse below $H$ the approximations given below lose their validity. Strictly speaking, the imposition of any positive $H$ barrier contradicts our previous assumption that the diffusion part of the dynamics are given by 
Heston-JtD. However, the extent of this contradiction can be made arbitrarily small by taking $H$ to be arbitrarily small. Furthermore, the existence of discrete tick sizes in practice implies that all of our approximations will be valid if we set $H$ equal to the lowest possible trading value (e.g. 0.01 USD or $0.01 \mathrm{EUR}$ ). In this case, we would then also have complete consistency with the discretized Heston-JTD model.

Let $X$ denote the process obtained by evaluating the natural logarithm of the scaled price process:

$$
X_{t}=\ln \left(S_{t} / H\right), \quad t>0 .
$$

We note that the power payoffs in the last section are exponential payoffs when expressed in terms of the random variable $X_{T}$ :

$$
Q\left(X_{T}, \lambda\right):=H^{-\lambda} \exp \left(-\lambda X_{T}\right)=\left(S_{T}\right)^{-\lambda},
$$

where we define $\lambda \equiv-p$ for later convenience.

\subsection{Gamma Payoffs}

Recall the well-known probability density function (PDF) of a Gamma distributed random variable with parameters $a>0$ and $b>0$, given by:

$$
f_{\text {Gamma }}(x ; a, b)=\frac{b^{a}}{\Gamma(a)} x^{a-1} \exp (-x b), \quad x>0 .
$$

We note that the mean of this gamma-distributed random variable is equal to $a / b$ and the variance is $a / b^{2}$. We note that a gamma distribution with an integer as $a$ parameter is also called an Erlang distribution. In what follows, we will only need Erlang distributions, but we refer to them as gamma distributions following common practice.

We introduce the concept of a family of Gamma-payoffs, whose definition depends on two parameters: a positive integer $n \in\{1,2, \ldots\}$ and a positive real number $z>0$. For each choice of $(n, z)$, the Gamma payoff occurs at a fixed maturity date $T$ and is related to the terminal log price relative $X_{T}$ by:

$\Gamma\left(X_{T} ; n, z\right)=f_{\text {Gamma }}\left(X_{T} ; n, n / z\right)=\frac{(n / z)^{n}}{\Gamma(n)} X_{T}^{n-1} \exp \left(-n X_{T} / z\right)$, if $X_{T}>0$ and zero otherwise.

In words, a Gamma-payoff is just the gamma PDF evaluated at the terminal log price relative $X_{T}$. The underlying gamma distribution has parameters $a=n$ and $b=n / z$, which translates into a mean equal to $z$ and variance $z^{2} / n$. Note that we assign zero gamma-payoffs for negative $z$ 's, which by our assumption can only arise through a jump-to-default.

To understand the behavior of Gamma payoffs, fix a number $z>0$. Then for each $n>1$, the Gamma-payoff corresponding to $(n, z)$ loosely resembles the payoff of a butterfly spread struck at $z$. As shown in Figure 1, the payoff is largest when $X_{T}$ finishes around $z$ for each fixed $n>1$. Moreover, the higher is $n$, the larger is the maximum payoff, but the faster is the decay from this maximum as $X_{T}$ moves away from $z$. This is also shown in Figure 1, where we have fixed $z=\log (100)$ and increased $n$ from 1 to 100 :

To appreciate how Gamma payoffs are related to the power payoffs of the last section, recall that $Q\left(X_{T}, \lambda\right):=H^{-\lambda} \exp \left(-\lambda X_{T}\right)$ denotes the payoff of a power claim when expressed in terms 


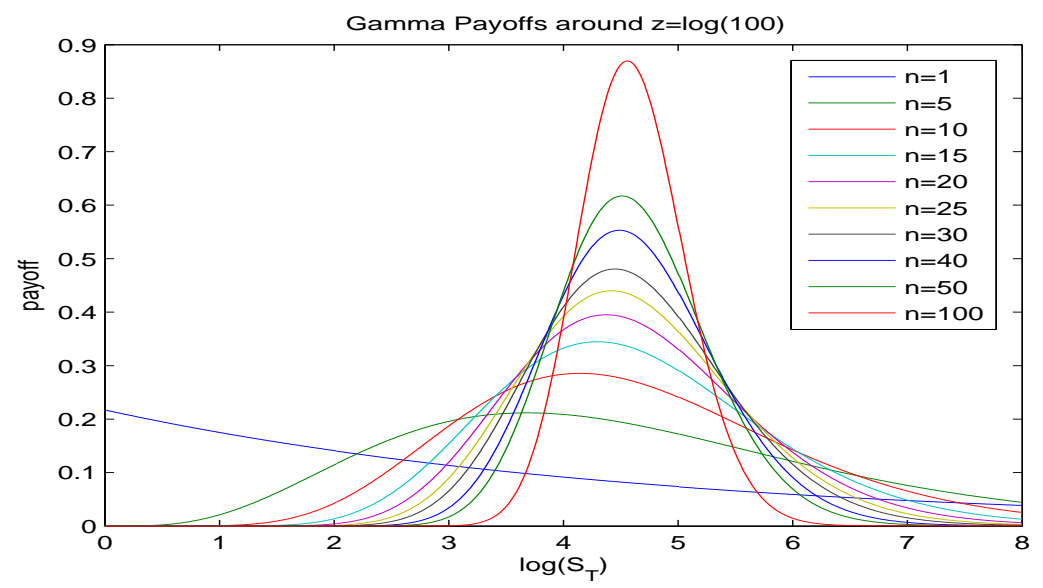

Figure 1: Gamma-payoffs $(z=\log (100))$

of the terminal $\log$ price relative $X_{T} \equiv \ln \left(S_{T} / H\right)$. The payoff function $\Gamma(x ; n, z)$ is related to the payoff function $Q(x, \lambda)$ in the following way:

$$
\Gamma(x ; n, z)=(-1)^{n-1} \frac{(n / z)^{n}}{\Gamma(n)} \frac{\partial^{n-1}}{\partial \lambda^{n-1}} Q(x, z / n):=L_{n, z}[Q](x, z / n), \text { for } z, x>0 .
$$

\subsection{Dirac Payoffs}

The Dirac payoff at $z$ is the limit of a Gamma-payoff for $n \rightarrow \infty$. It depends on a parameter $z>0$ and pays out:

$$
G O D\left(X_{T} ; z\right)=\lim _{n \rightarrow \infty} \Gamma\left(X_{T} ; n, z\right)= \begin{cases}\infty & \text { if } X_{T}=z ; \\ 0 & \text { else. }\end{cases}
$$

In other words, the payoff is infinite if $X$ ends at $z$, which unfortunately for the buyer occurs with zero probability (sorry to disappoint). Although the probability of receiving a nonzero payoff from a Dirac payoff is zero, the initial cost of replicating a Dirac payoff is positive and is also finite except right at expiry. As a result, we have that the time zero-price of a Dirac payoff is given by:

$$
\begin{aligned}
h(z) & =\exp (-r T) E_{Q}\left[G O D\left(X_{T} ; z\right)\right] \\
& =\exp (-r T) \lim _{n \rightarrow \infty} E_{Q}\left[\Gamma\left(X_{T} ; n, z\right)\right] \\
& =\exp (-r T) \lim _{n \rightarrow \infty} \int_{-\infty}^{+\infty} \frac{(n / z)^{n}}{\Gamma(n)} x^{n-1} \exp (-n x / z) f_{X_{T}}(x) \mathrm{d} x \\
& =\exp (-r T) f_{X_{T}}(z) .
\end{aligned}
$$

We note that the above argument is based on the Post-Widder Laplace Inversion formula.

The nice thing about Dirac payoffs is that they can be used to hedge any payoff, which depends only on the final $(\log )$ stock price. Indeed suppose you have a European Call option maturing 
at time $T$ and with strike $K$. For convenience, set $H=1$ and let $k:=\log K$. The argument that follows only holds for $k>0$. Then holding the appropriate continuum of Dirac payoffs is equivalent to holding the call. Indeed, if for each real $x$, we have an infinitessimal position given by $\left.(\exp (x)-\exp (k))^{+}\right) d x$ units of each Dirac claim struck at $x$, then for all $0 \leq t \leq T$, this portfolio has the same value as a call since:

$$
\begin{aligned}
E C_{t}(T, K) & =\exp (-r(T-t)) E_{Q}\left[\left(\exp \left(X_{T}\right)-\exp (k)\right)^{+} \mid \mathcal{F}_{t}\right] \\
& =\exp (-r(T-t)) \int_{-\infty}^{+\infty}(\exp (x)-\exp (k))^{+} f_{X_{T} \mid \mathcal{F}_{t}}(x) \mathrm{d} x \\
& =\int_{-\infty}^{+\infty}(\exp (x)-\exp (k))^{+} \exp (-r(T-t)) E_{Q}\left[G O D\left(X_{T} ; x\right) \mid \mathcal{F}_{t}\right] \mathrm{d} x
\end{aligned}
$$

Furthermore, the hedge portfolio of each Gamma claim can be easily derived from the known hedge portfolio of the corresponding power claim. Suppose that the hedge of the power payoff $Q\left(X_{T}, \lambda\right)$ requires a position at some time $t \in[0, T]$ of say $\phi_{t}^{(i)}\left(X_{t}, \lambda\right)$ units of assets $A_{i}$, for $i=1, \ldots, N$. Then one can also hedge the Gamma payoff $\Gamma\left(X_{T} ; n, z\right)$ by dynamically trading the assets $A_{i}, i=1, \ldots, N$. At each time $t \in[0, T]$, the required position in asset $A_{i}$ is given by:

$$
\psi_{t}^{(i)}\left(X_{t} ; n, z\right)=L_{n, z}\left[\phi_{t}^{(i)}\right]\left(X_{t}, z / n\right) \text {, for } z, x>0 .
$$

In Figure 2, we show the call payoff function together with its approximation by a portfolio of Gamma-payoffs.

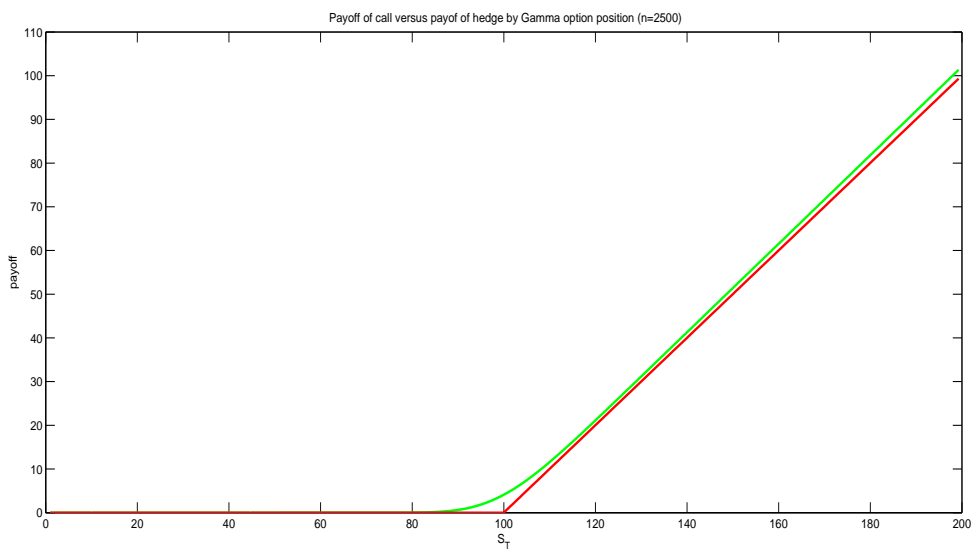

Figure 2: Vanilla payoff vs payoff a portfolio of gamma options

\section{Orthogonal Polynomial Approximation Hedge}

In the last two sections, we have shown that an arbitrarily given path-independent payoff be replicated via dynamic trading in stock, bond, VS, and CDS. Our approach used the uncountably 
infinite family of Dirac payoffs of different strikes as a basis. The ability to dynamically replicate path-independent payoffs motivates the problem of finding alternative families of basis functions. While any infinite family of basis functions will provide a perfect hedge, it is of interest to compare different finite families in terms of their speed of convergence to the target. In this section, we will use the theory of orthogonal polynomials to approximate the target payoff by a finite family of polynomials. For some references to the theory of orthogonal polynomials (and especially the link with stochastic processes), we refer the reader to [4]).

Let us first focus on the simplest situation, where one has a uniform weight measure. Suppose that we wish to replicate a target payoff $f\left(S_{T}\right)$ over a reasonable interval of possible final stock price values, say $[a, b]$. The best approximation of the payoff function $f(x)$ by a polynomial of degree $m, P_{m}^{*}(x)$ can be found in the following way. Let us denote by $\mathcal{P}_{m}$ the set of polynomials (over the real line) of degree at most $m$. The general theory of orthogonal polynomials says that:

$$
\min _{P_{m}(x) \in \mathcal{P}_{m}} \int_{a}^{b}\left[f(x)-P_{m}(x)\right]^{2} \mathrm{~d} x,
$$

is given by:

$$
P_{m}^{*}(x)=\sum_{i=0}^{m} b_{i} q_{i}(x),
$$

where $q_{i}(x)$ is the orthonormal polynomial of degree $i$ on $[a, b]$ with respect to the uniform measure. The polynomial $q_{i}(x)$ can be written in terms of the Legendre polynomial of degree $i$ which are orthogonal with respect to the uniform measure on $[-1,1]$. The coefficients $b_{i}$ are given by

$$
b_{i}=\int_{a}^{b} f(x) q_{i}(x) \mathrm{d} x, \quad i=1, \ldots, m .
$$

For example, suppose that $S_{0}=100$ and we want to approximate the payoff of an at-the-money call by a polynomial of degree $m=6$ over the stock price interval $[0,300]$. We obtain:

$$
\begin{aligned}
\left(S_{T}-100\right)^{+} \approx & -5.23012035469737+0.87924498488373 S_{T}--0.03203881682890 S_{T}^{2}+0.00041002420953 S_{T}^{3} \\
& -0.00000211419402 S_{T}^{4}+0.00000000501164 S_{T}^{5}-0.00000000000452 S_{T}^{6} .
\end{aligned}
$$

In Figure 3, we graph the call payoff function together with its approximation for $m=6$.

In Figure 4, we graph several approximations for different values of $m$.

The theory can be generalized to non-uniform weight measures $w(x)$ with respect to which we seek the best polynomial approximation. Thus, the more general problem is:

$$
\min _{P_{m}(x) \in \mathcal{P}_{m}} \int_{C}\left(f(x)-P_{m}(x)\right)^{2} w(x) \mathrm{d} x,
$$

where we are now integrating over a region $C$ with respect to the weights $w(x)$. The previous case corresponded to $C=[a, b]$ and the uniform weight $w(x)=1$. In the general case, the best fitting polynomial of degree $m$ is given by:

$$
P_{m}^{*}(x)=\sum_{i=0}^{m} b_{i} Q_{i}(x), \quad b_{i}=\int_{C} f(x) Q_{i}(x) w(x) \mathrm{d} x, \quad i=1, \ldots, m
$$




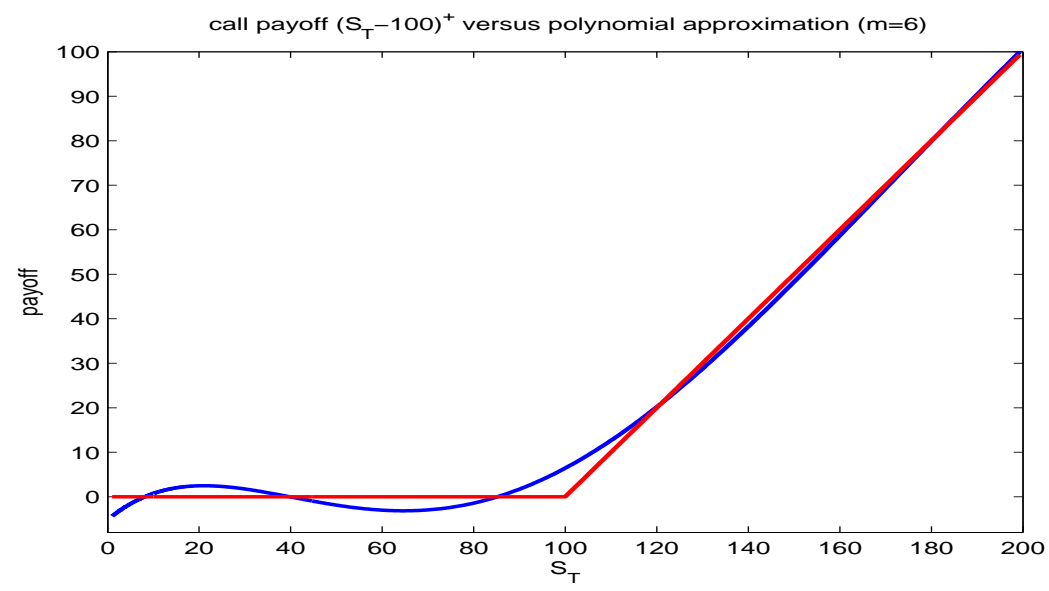

Figure 3: Vanilla payoff vs polynomial approximation

where $Q_{i}(x)$ is the orthonormal polynomial of degree $i$ with respect to $w(x) \mathrm{d} x$ :

$$
\int_{C} Q_{i}(x) Q_{j}(x) w(x) \mathrm{d} x=\delta_{i j} .
$$

\section{Summary and Extensions}

Assuming that the stock price dynamics are given by HestonJTD, we first showed that the payoffs to a static position in a power claim could be replicated via dynamic trading in stock, bond, VS, and CDS. We then showed that the ability to take static positions in power claims of all real powers was equivalent to the ability to take static positions in Dirac payoffs of all strikes. This motivated the construction of families of orthogonal polynomials, which form an alternative basis. No matter which basis was chosen, we were able to give explicit formulas relating the position in each basis asset to the state variables in the HestonJTD model.

Future research can focus on extending the dynamics under which perfect replication is possible, most likely by allowing positions in additional hedge instruments. If one does not wish to expand the set of hedging vehicles, one can also explore super and sub-replicating strategies along with the associated no-arbitrage bounds on values. In the interests of brevity, these extensions are best left for future research.

\section{References}

[1] Heston, S. (1993): A closed-form solution for options with stochastic volatility with applications to bond and currency options, Review of Financial Studies 6, 327-343.

[2] Miller, K. S., Bernstein, R. I. and Blumenson L. E. (1958): Generalized Rayleigh processes, Quarterly of Applied Mathematics 16, 137-145. 


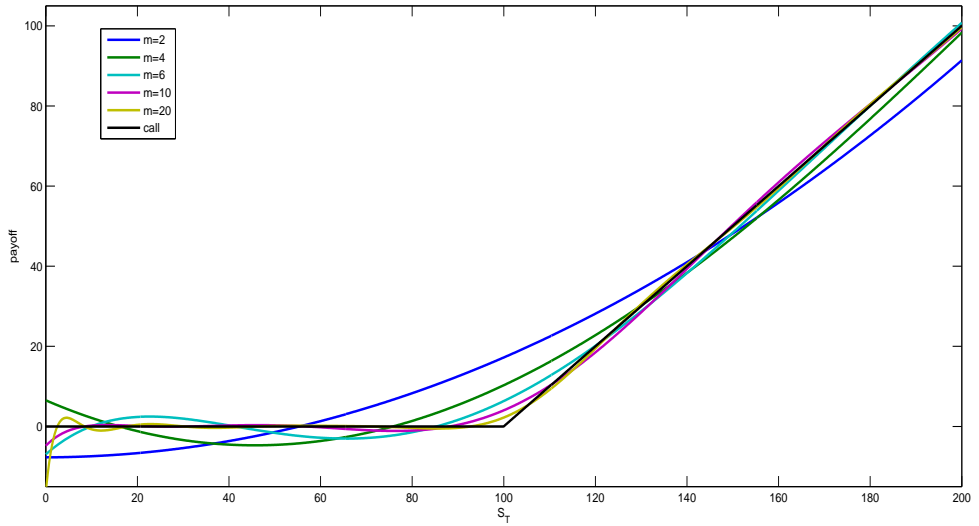

Figure 4: Vanilla payoff vs polynomial approximation $m=2,4,6,10,20$.

[3] Schoutens, W., Simons E. and Tistaert, J. (2004): A perfect calibration! Now what ?, Wilmott Magazine, March 2005, 66-78.

[4] Schoutens, W. (2000) Stochastic Processes and Orthogonal Polynomials. Lecture Notes in Statistics 146. Springer-Verlag, New York.

[5] Widder, D.V. (1941) The Laplace Transform, Princeton University Press, Princeton, New Jersey, 1941. 